\title{
Gradhiva
}

Revue d'anthropologie et d'histoire des arts

$14 \mid 2011$

Carl Einstein et les primitivismes

\section{Exposition de statuettes de bronze antiques antérieures à notre ère (art hittite, étrusque, égyptien, grec), 1933}

\section{Carl Einstein}

Traducteur : Isabelle Kalinowski

\section{OpenEdition \\ Journals}

\section{Édition électronique}

URL : http://journals.openedition.org/gradhiva/2208

DOI : 10.4000/gradhiva.2208

ISSN : 1760-849X

\section{Éditeur}

Musée du quai Branly Jacques Chirac

\section{Édition imprimée}

Date de publication : 30 novembre 2011

Pagination : 234-251

ISBN : 978-2-35744-046-3

ISSN : 0764-8928

\section{Référence électronique}

Carl Einstein, «Exposition de statuettes de bronze antiques antérieures à notre ère (art hittite, étrusque, égyptien, grec), 1933 », Gradhiva [En ligne], 14 | 2011, mis en ligne le 30 mai 2012, consulté le 30 avril 2019. URL : http://journals.openedition.org/gradhiva/2208; DOI : 10.4000/gradhiva.2208 


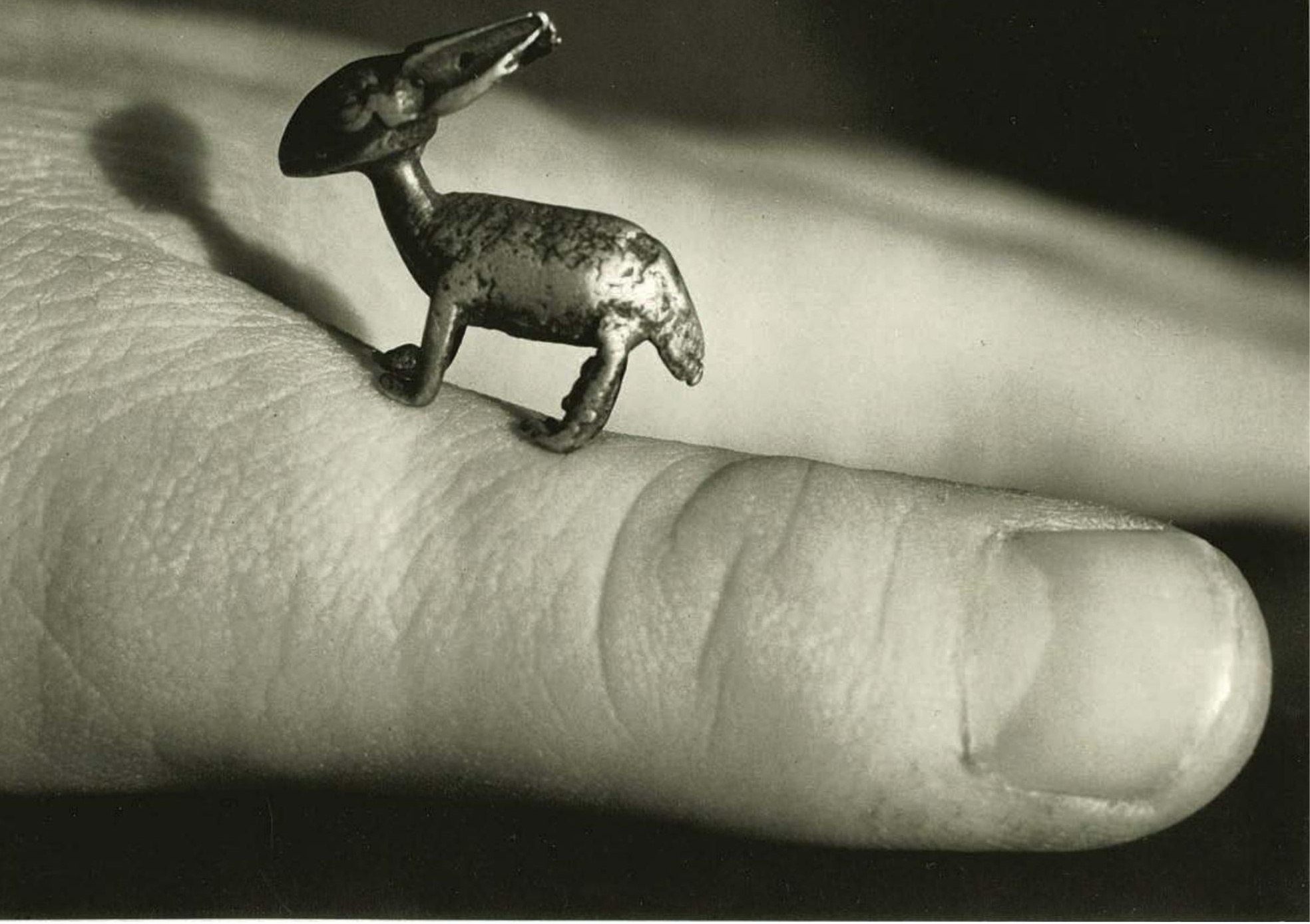

Fig. 1 Man Ray, Goldweight Akan, v. 1933, coll. part. Paris @ MAN RAY TRUST / ADAGP, Paris 2011. 


\title{
1933 Exposition de statuettes de bronze antiques antérieures à notre ère (art hittite, étrusque, égyptien, grec)
}

\begin{abstract}
Exposition de statuettes de bronze antiques antérieures à notre ère est le seul texte de Carl Einstein que nous possédions sur l'art de la haute Antiquité orientale et méditerranéenne; un travail de commande, composé en 1933 à la requête des frères Stora pour leur galerie newyorkaise, 670, Fifth Avenue. L'exposition regroupait principalement des œuvres de la collection particulière des frères Stora, qui possédaient également une galerie à Paris, 32, boulevard Haussmann ${ }^{1}$. D'abord rédigé en allemand, ce texte fut traduit pour l'occasion en anglais sous le titre Exhibition of Bronze Statuettes B.C. (Hittite, Etruscan, Egyptian, Greek) et ne parut que sous cette forme. La présente traduction de ce document jusque-là inédit en français a été établie à partir du manuscrit allemand conservé au Carl Einstein Archiv de Berlin et édité dans le tome IV de ses œuvres complètes. Un commentaire de ce texte est proposé par Isabelle Kalinowski dans son article "Les trois moments de Carl Einstein", p.100-121. Les notes ont été établies avec la collaboration de Frédéric Junqua.
\end{abstract}

L'histoire est une projection du présent vivant; la sélection et l'évaluation des époques est déterminée par le courant et la structure de l'aujourd'hui. L'homme angoissé par la mort veut trouver dans le passé la garantie de sa durée et il veut justifier et ancrer dans le retour du passé le présent vite englouti.

La primitivisation de l'art actuel est manifeste. L'homme itinérant d'aujourd'hui, possédé par des forces dynamiques, cherche à nouveau une patrie durable et se place sous la protection des formes tectoniques stables. On se défend contre le chaos irrémédiable et contre la multiplicité des possibilités en adoptant une discipline plus stricte, en sélectionnant des formes et des symboles. C'est à cette tendance que répond l'actuelle standardisation des types humains et des choses qui ont pour fonction de servir.

Contre l'utopie optimiste et vaine d'une évolution linéaire, nous posons la loi de la régression périodique; les couches psychiques inutilisées percent à nouveau et ramènent l'existence et l'action dans un courant souvent contraire. Le savoir acquis devient inefficace d'un point de vue psychique. Il n'est plus en mesure de susciter les chocs qui nous sont nécessai- res; la conscience signale qu'un certain type de processus psychiques s'est adapté, mécanisé, et qu'il est devenu prévisible. On peut remarquer que la science est un savoir secret et que son succès tient pour une part à ce qu'on ne la comprend pas. Mais ces îlots de savoir surnagent à peine de la masse obscure des processus irrationnels. Les éléments qui peuvent être saisis sur un mode logique ou concret ne sont que des points d'arrêt, des reflets à la surface d'un courant qui nous emporte mystérieusement; on se rend compte alors qu'une part considérable des processus psychiques vont à l'encontre de la logique ou sont extérieurs à elle et qu'on se fonde plus souvent sur des analogies mythiques que sur des raisonnements logiques. Ces processus ne peuvent être saisis et fixés, dans un premier temps, que métaphoriquement, c'est-à-dire dans des signes.

Cette attitude antinaturaliste est une marque d'archaïsme caractérisé. Ces couches psychiques anciennes possèdent des caractéristiques collectives; elles favoriseront donc le recours à des formes collectives, c'est-à-dire tectoniques. Dans le même temps, le fossé entre la réalité mécanisée et l'automatisme psychique 
ne cesse de se creuser et on est ainsi conduit à créer librement, par-delà la reproduction de l'existant, des formes et des processus mythiques. Contre cette percée dangereuse des hallucinations, on se protège en cherchant des formes précises, afin d'endiguer le flot des visions. En même temps, le caractère collectif des couches dès lors activées et précédemment refoulées impose le recours à des signes validés collectivement, qui compensent (équilibrent) le vécu subjectif et isolé. On pourrait parler d'un archaïsme psychique. En modifiant ainsi les prémisses, on impose une nouvelle sélection du donné historique. En fixant des processus qui ne correspondent pas à ceux qui se déroulent mécaniquement, on rompt avec les naturalismes, ou on les détruit. Ces processus apparemment dépourvus de sens lorsqu'on les évalue d'un point de vue logique vont appeler une expression mythique, c'està-dire qu'on va nécessairement créer des formes qui ne sont pas attestées par des modèles. Les processus psychiques présentent dès lors une structure difficile à saisir d'un point de vue logique; la simplification heuristique qui, ni plus ni moins, exclut le concret vivant, fait place à une contrainte de métamorphose, et l'unité des signes se fonde désormais sur le drame de la métamorphose. À la place de l'observation des faits s'affirme dès lors la fatalité du destin; on interprète les processus impossibles à saisir d'un point de vue rationnel par l'hallucination et les signes.

La science, presque incompréhensible sinon pour les féticheurs, cette science à laquelle on accorde foi avec perplexité et embarras, n'est que l'affaire d'un très petit nombre et ne produit presque aucun effet. Celui qui est prétendument éclairé porte en lui, obscure et confuse, une vague image mythique du monde qu'il préfère dissimuler pudiquement. Mais ces représentations fausses au sens scientifique sont plus lourdes de conséquences et d'effets que toutes les connaissances qui ne sont que des erreurs non encore démasquées. Ces délires enfantins sont coiffés par quelques formes universelles qui suscitent l'admiration, mais dont la vérité provisoire peut être détruite demain. C'est justement la forme incompréhensible des formules scientifiques, ces tabous d'aujourd'hui, qui confère à cellesci leur énigmatique influence.

De la même façon, l'art s'est volatilisé dans un brouillard vague, et les œuvres s'enlisent dans le marais des paraphrases. Des prophètes évoquent la force de rétention d'un rouge et expliquent comment ce dernier suscite un bleu serein dans le coin droit. Avouons-le sans détours, l'art a incroyablement perdu de sa puissance spirituelle, parce qu'il n'est plus affecté au service d'un ordre puissant et strict. L'art seul n'est pas en mesure d'en créer un, et les critères exclusivement esthétiques ou formels traduisent précisément la pauvreté spirituelle d'une grande part des images actuelles.

Les œuvres de la haute Antiquité étaient au service d'une image du monde magique et dotée d'une signification et elles étaient par là vouées à la conservation des dieux et du peuple; elles étaient une projection des mythes dogmatiques et demeuraient subordonnées aux forces spirituelles et sociales primaires. Une statue représentait alors bien davantage qu'une imitation ou une création de formes isolée; elle était au service d'objectifs de première importance, à savoir la préservation et le renforcement de la vie. Ainsi, pour les Égyptiens, le verbe sculpter était tout simplement synonyme de "donner vie». Les sculptures étaient le contenant et la prison des esprits et des dieux, elles immobilisaient magiquement la substance vivante du défunt, son $k a$. Cet art-là, reconnaissons-le, n'avait pas la prétention de constituer une sphère autonome à partir de tubes de peinture et de vagues esthétismes. L'œuvre imagée n'était regardée que comme un outil pour ceux qui croyaient aux forces magiques auxquelles la perpétuation du monde et de l'homme était attachée comme à un destin; l'art était le témoin d'une existence de piété et il possédait une signification parce qu'il servait, par ses signes, une vision du monde et un culte dont dépendaient l'existence et le destin d'un peuple. Les modalités et la démarche de cet art d'évocation de la haute Antiquité n'étaient pas déterminées par des esthétismes mais par des objectifs plus importants, extérieurs à l'art.

Dans cet esprit des premiers temps antiques, l'artiste était ordonné dans un tout dont il avait à être l'auxiliaire, au service de la croyance et de la société. L'art était nécessaire pour ancrer les dieux dans un pays et pour repousser les esprits malfaisants. Pour les Grecs, déjà, la haute Antiquité devint vite énigmatique; car si les artistes impies étaient alors proscrits, cette époque ne connut pas de lettrés au sens d'Hérodote ou de Catulle. Le succès illimité de l'Antiquité tardive - disons plutôt de la modernité grecque - ne se fonda pas seulement sur ses œuvres d'art mais surtout sur la montée en puissance des lettrés et des philosophes savants. Ce ne sont ni Phidias ni les hurlements d'Achille qui ont sauvé la Grèce d'un oubli sans retour, mais les définitions d'Aristote qui, avec le syllogisme, a forgé un outil de portée internationale, ou Hérodote, qui a réussi à démontrer que, par le savoir et 


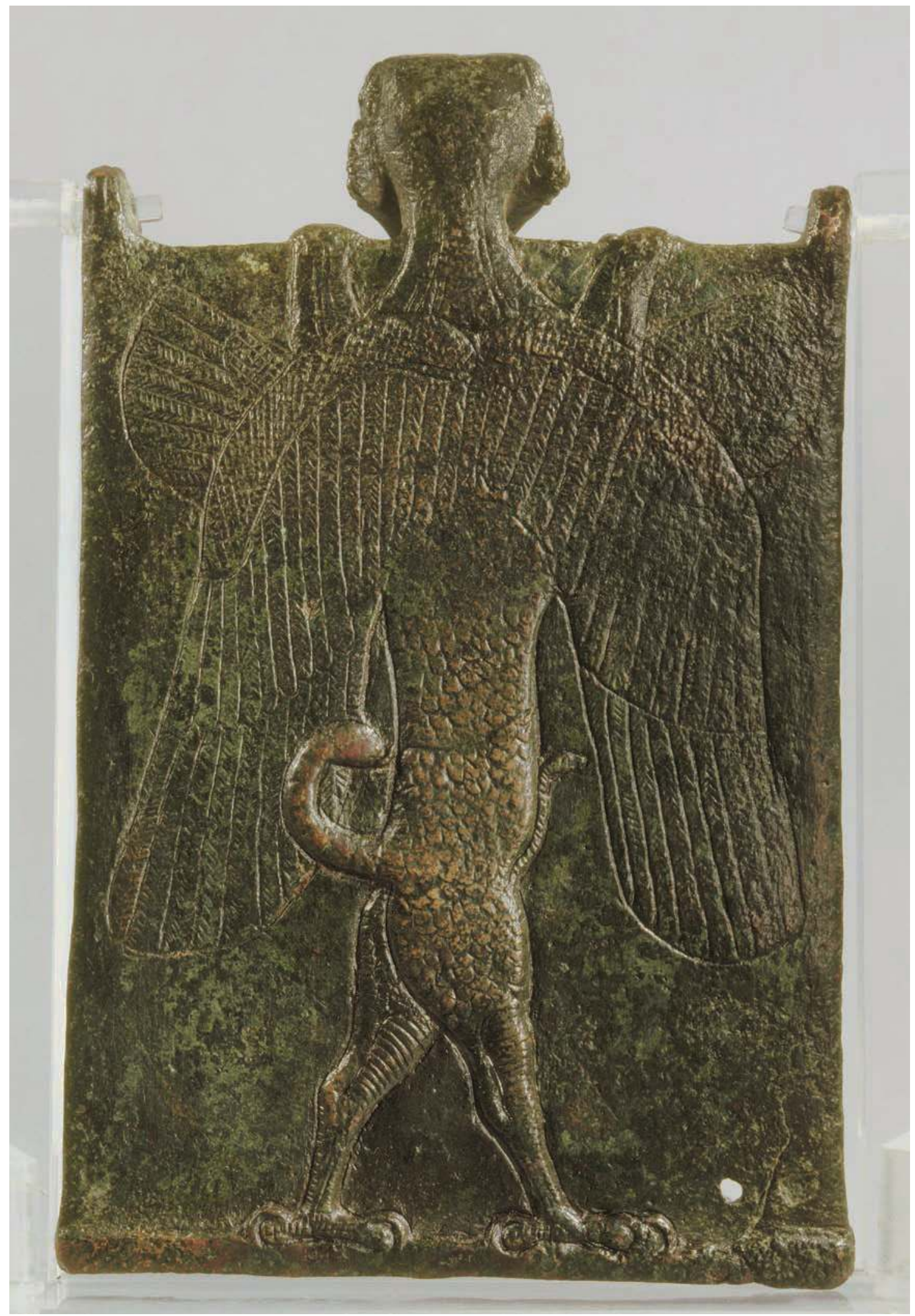

Fig. 2 Plaque de conjuration contre la démone Lamashtu, site d’Arslan Tash, vers 750 av. J.-C., Paris, musée du Louvre @ RMN / Les frères Chuzeville. 


\section{TEXTES DE CARL EINSTEIN}

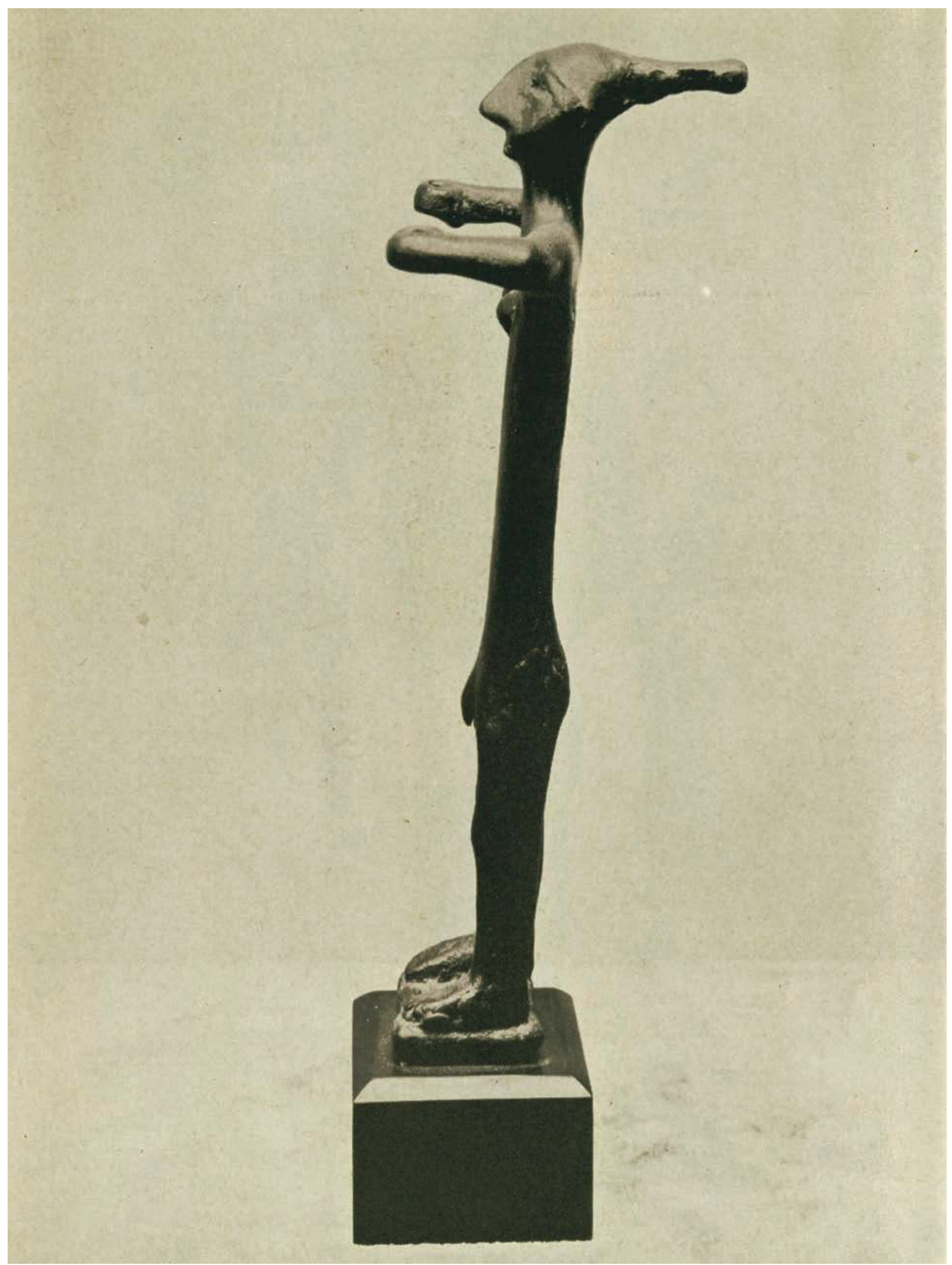

Fig. 3 Figure hittite, vers 2000 av. J.-C., planche 4 du catalogue préfacé par Carl Einstein de l'exposition de statuettes de bronze antiques à la galerie Stora, New York, 1933. 
la critique, l'individu pouvait dépasser la tradition de son peuple et prendre possession du passé et de la terre.

Avouons-le : l'école a menti, comme Homère. La Renaissance, qui a été une composante du processus de la Réforme, a livré une Antiquité de substitution, l'Antiquité romaine, et l'a opposée à la scolastique médiévale et à l'orthodoxie byzantine. Ensuite, le xvIII ${ }^{\mathrm{e}}$ siècle éclairé a eu à nouveau recours à l'Antiquité pour bâtir, en coulisse, une utopie élégante et par trop confortable de la perfection sereine. Cette Antiquité disons goethéenne appréciait par-dessus tout les exemples rhétoriques et maniéristes de décadence. Un renversement de ces représentations académiques en stuc a été accompli plus tard par le puissant Hölderlin et par Nietzsche. Nietzsche dépeint l'époque fameuse de Socrate, Platon et Périclès comme une époque de lamentable déclin.

L'Orient s'étendait sous le regard de nos pères comme un marais immobile et inerte. Depuis les guerres contre les Perses, l'Asiatique apparaissait en gros comme l'ennemi classique de l'homme du continent, et la Grèce couvrait de son ombre fatale l'Orient, son ancêtre. Cependant, celui-ci n'avait jamais cessé de menacer la jeune culture continentale. Nous connaissons cette vengeance lente et à chaque fois inexorable de l'Orient ancien depuis les conquêtes d'Alexandre. Les Romains se plièrent aux cultes d'Asie Mineure; puis le christianisme s'imposa à l'Europe. La Syrie, l'Asie Mineure et Byzance dominèrent l'art médiéval. Dans la gnose, la liturgie et la symbolique de l'Orient ancien se réveillèrent, et les religions astrales de l'Orient chenu retentissaient à nouveau de ciels mystérieux.

Cet art de l'Asie Mineure dans la haute Antiquité est, de façon générale, un art typique de bâtisseurs de villes et de colonisateurs; il atteint déjà la maturité d'un grand art au IVe millénaire.

Le cosmos a la stabilité d'une création définitive; lui et les hommes sont les œuvres d'art des dieux. L'homme est fait de terre et du sang des dieux; ainsi, les Égyptiens, à la mentalité tectonique, qualifiaient leur dieu de "maçon de l'homme». La survie de l'homme est garantie par la permanence de l'être des dieux, que rien ne vient troubler, et par la régularité imperturbable du cours des astres; car le dieu et l'astre ne font qu'un. L'astronomie est importante en tant que témoignage et observation des dieux et de leurs agissements; l'astrologie était de part en part une théologie. Marduk, Ishtar, Tammuz, etc. sont aussi des astres. Pour que le monde ne retombe pas inexorablement dans le chaos, les astres doivent poursuivre leur course, imperturbables. On s'approche d'eux, pieusement, en élevant des tours à étages qui sont à l'image de la montagne-univers dressée vers les sphères célestes, ou bien on construit dans les plaines les pyramides, demeures éternelles des morts. Aujourd'hui encore, les Arabes disent d'elles : «Le temps est craint de tous, mais le temps a peur de la pyramide. "

Les Hittites semblent avoir eu une conception du monde apparentée à celle des Babyloniens. Ils vénéraient une sorte d'Astarté, la Grande Mère; dans la Komana (Cappadoce), elle s'appelait Mâ, la déesse à la couronne murale. Elle était servie par les Galles, des prêtres eunuques, et par des prêtresses appelées amazones. Un oiseau, la colombe sans doute, était son animal sacré (voir les représentations du Saint-Esprit sous la forme d'une colombe, et le verset de la Bible où il est dit que "l'esprit de Dieu flottait au-dessus des eaux»). Tammuz, le dieu assassiné, le Dionysos des Grecs, faisait l'objet de cultes extatiques. Le dieu suprême était Teshub, le dieu des éclairs et de l'orage; chez les Hittites occidentaux, il s'appelait Tarku; la Bible évoque ses grondements de tonnerre. Dans la tribu hittite des Mitanni, on priait la déesse Shaukas, qui correspondait en tout point à Ishtar. Dans les tribus du lac de Van régnait une triade de divinités semblable à la triade babylonienne ancienne, avec un dieu du soleil, Ardis, qui était apparenté au Marduk de Babylone.

Les événements qui avaient lieu sur la terre correspondaient aux événements célestes. Un texte théologique de Babylone enseigne: "Il en est ici sur la terre comme là-haut; car ce qui est ici sur la terre est à l'image de ce qui est au firmament. » En un sens, le cosmos des hommes de la haute Antiquité était plus étendu que le nôtre, car il comprenait les domaines transcendants du ciel et du monde souterrain; une géographie mythique. L'homme est un microcosme et il vit comme l'ombre des dieux. Les parties de son corps possèdent une signification symbolique et magique. L'homme gagne en réalité en se rapprochant des dieux par la prière et l'accomplissement des rites : les temples sont à l'image du ciel et ils ne peuvent donc être dessinés que par ceux qui ont le savoir des dieux. Ainsi, Gudéa de Tello affirme que les plans de ceux qu'il a fait bâtir lui ont été révélés par les dieux en rêve. Sa déesse protectrice Nisaba «lui ouvre l'esprit » et lui enseigne la signification des nombres; "Elle tenait à la main le pur stylet d'écriture, elle portait une tablette sur laquelle était inscrite la constellation favorable, et elle réfléchissait à tout cela.» 
Ces cultures, de façon générale, se présentent comme des cultures urbaines achevées. L'État est de type proprement théogénique. Le roi est le représentant et l'intermédiaire des dieux; le pouvoir de l'État et celui du roi-prêtre sont étroitement liés. Les Babyloniens regardaient leur roi comme une image de Marduk; Gudéa s'intitule "dieu de son pays", tandis qu'un autre roi, Hammurabi, se fait appeler " roi-dieu de la ville ", « dieu du soleil ", etc. Les témoignages des archives hittites de Boghazkoï rapportent que des sacrifices étaient déposés au pied des statues royales, et un texte recommande de sacrifier devant l'image du roi Hattushil. Le roi du Mittani Tushratta écrit à Aménophis IV qu'il s'est «installé comme un dieu sur le trône de son père ». Voir le visage du roi est appelé un acte de "vie».

Les astres qui sillonnent le ciel au-dessus de ces sédentaires suivent des voies toutes tracées, les dieux eux-mêmes sont devenus sédentaires, ils résident dans les temples. Temples et citadelles sont le centre et le siège du pouvoir et du savoir. Le clergé gère la propriété de la terre.

Un art se développe alors qui est entièrement dominé par l'architecture. On ne prie plus dans des bosquets, la religion devient tectonique; une théologie dogmatique est créée. Les esprits élémentaires errants des nomades itinérants se fixent pour devenir des dieux personnels, le chaos des forces difficilement intelligibles s'ordonne en un cosmos, le calendrier devient une des bases de l'existence et des événements; au demeurant, le déroulement de l'année s'accomplit sur un mode magique, et toute réussite est subordonnée aux sacrifices, à l'exécution réglée des rites et à des incantations. On a abandonné la vie des nomades, des chasseurs et des bergers qui se déplacent sans trêve; les hommes séjournent à présent dans l'ombre protectrice des dieux de la ville.

Les sédentaires ont besoin de stabilité; la foi donne par conséquent l'intuition des lois du cours des astres; le caractère répétitif des événements est mis en avant; des rites sont établis, un canon d'images fait son apparition, et il constitue une partie du rituel. Par le dogme et le rite, on réprime toutes les hallucinations subjectives; car elles privaient les images de leur efficacité magique. L'art est créé par les prêtres, un monde dogmatique.

Ces villes sont séparées par de vastes territoires inoccupés. Dans leur proximité résident les morts. Leurs murailles ne cessent d'être assaillies par des peuples barbares et impies qui se déplacent sans trêve entre les zones de pâturage, ou, en quête de meilleures terres, sont attirés par les riches cités. La menace de la primitivisation est donc toujours présente, et des éléments nomades s'imposent peut-être dans l'art urbain tectonique, une mobilité graphique. Les villes s'étendent, bruissantes, sous la protection du dieu de la ville; quand une cité est conquise, on peut identifier le dieu vaincu avec le sien propre, pour renforcer sa puissance magique, ou bien traîner jusqu'à la ville le dieu soumis, afin que les vaincus se placent sous la protection du dieu vainqueur.

On est loin, ici, du réalisme agité du chasseur, qui veut prendre possession des animaux dangereux et plus forts que lui en reproduisant leur image, de la même façon que, en appelant quelqu'un par son nom, on le place sous son emprise; car l'image et le nom sont des doubles, des parties de la substance vivante ou de celle de l'âme.

Désormais se développe le style des hommes des forteresses et des maisons. Au monde des hommes s'opposent celui des dieux et un autre monde angoissant, celui des morts. On enferme les dieux comme les rois dans des statues que l'on évoque par des prières; ainsi, les Égyptiens appellent les statues «la demeure du $k a$ ». Les dieux et les souverains ou leur double habitent dans les statues, et ces effigies sont entretenues et nourries; car elles sont le signe vivant et efficace des dieux et des rois; c'est grâce à l'effigie que ces derniers résident dans un lieu fixe et demeurent accessibles. La statue n'est donc pas un amusement esthétique inoffensif, mais une personne vivante, qui agit de façon miraculeuse. Des miracles et des menaces émanent d'elle. Par ce sortilège, on fixe les dieux dans les temples et les statues; car ceux-ci sont les garants de la survie des hommes. L'efficacité magique d'une image, et par conséquent sa possession, sont une question de vie ou de mort pour la ville et le peuple. Ces images des dieux et des morts doivent être "maintenues en vie", et Gudéa fait inscrire sur sa statue : "Que sa vie perdure." C'est en ces termes que les Babyloniens pieux désignaient les statues, et les Égyptiens appelaient par exemple une statue de Thoutmosis III statue «de millions d'années ». Les statues sont donc les signes de la durée, et un art statique et tectonique est par conséquent requis. On enferme dans les statues les dieux mauvais et leurs forces méchantes; de même, on confine les esprits des morts dans des tombeaux qui sont une bonne garantie, tout comme les chasseurs recouvraient de pierres les cadavres des animaux ou les enroulaient dans des fibres d'écorce et des lacets.

Fig. 4 Passe-guide orné d'une scène de domptage, Turquie, Boghazkoï ?, vers 2500 av. J.-C., Paris, musée du Louvre @ RMN / Franck Raux. 


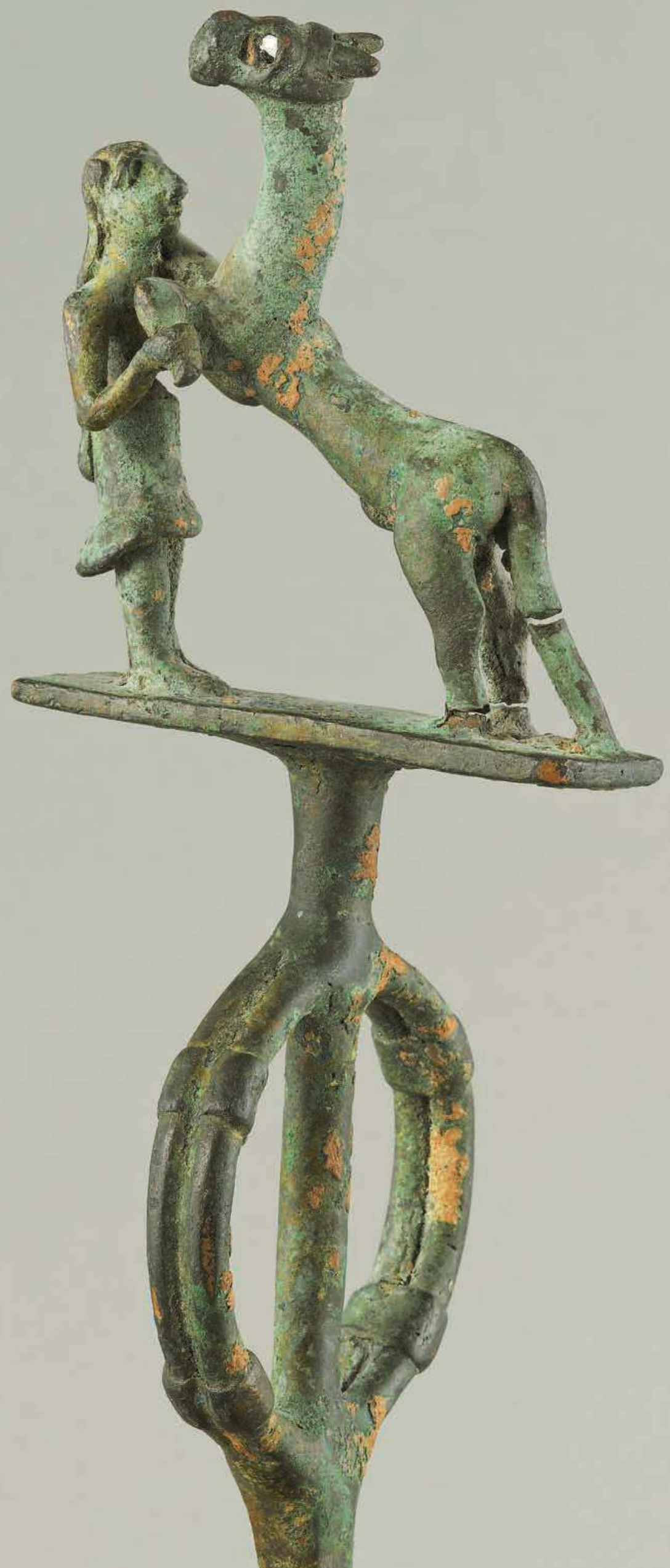




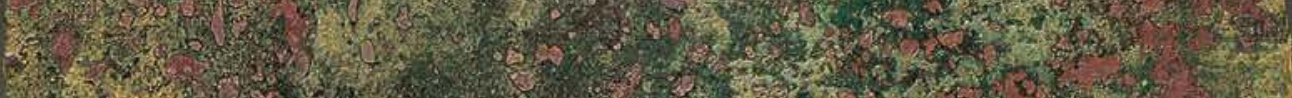

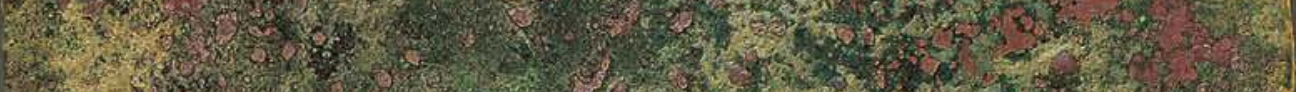

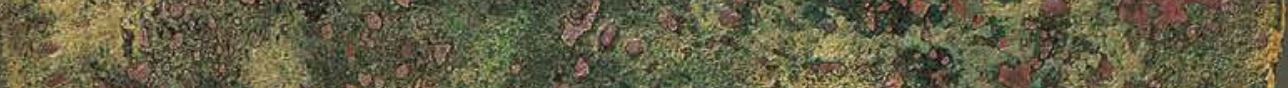

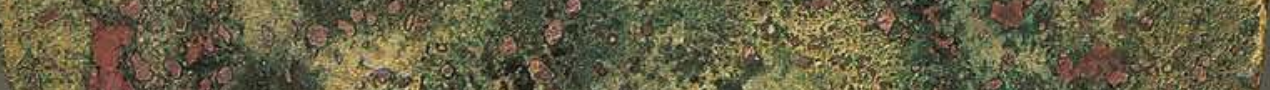

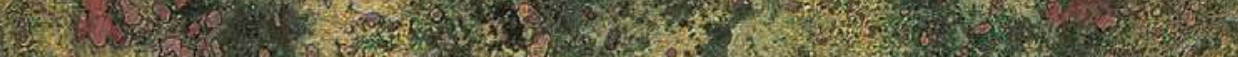

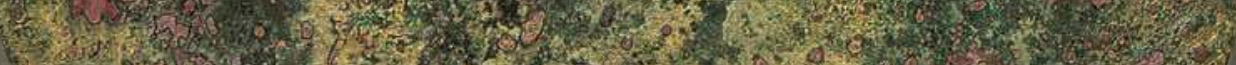

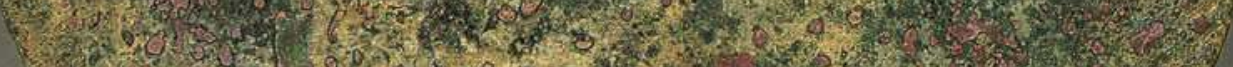

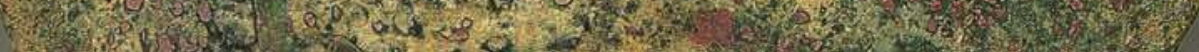

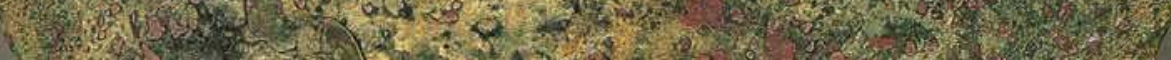

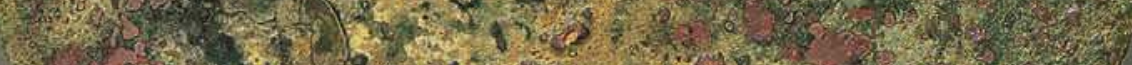

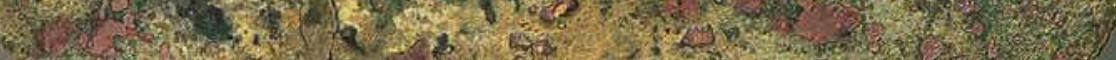

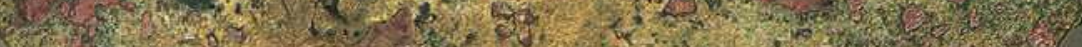
(1)

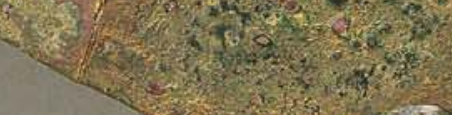

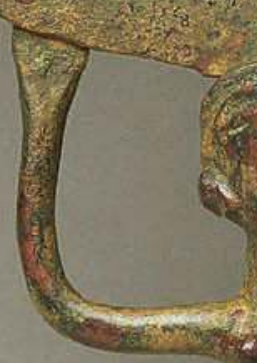

320

ex 6

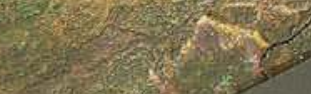


L'homme angoissé par la mort s'efforce de préserver la vie dans l'effigie, et le sédentaire marqué par l'angoisse du chaos a recours aux formes tectoniques. La survie des défunts est liée à celle des formes corporelles; voilà pourquoi on aime la pierre, qui ne bouge pas. C'est cet état d'esprit qui s'exprime dans la formule égyptienne: "Que les montagnes immuables abritent des monuments. " On peut dire que la statue est la demeure de la substance magique.

L'art animiste des chasseurs est de type dramatique, il reflète l'errance sans trêve. Les esprits errants, la nature inquiétante et peu ordonnée. Les sédentaires et les hommes des villes créent un art conservateur qui est, de surcroît, soumis aux conventions de l'éternel, à l'exigence de permanence et de durée. On s'éloigne alors du vieux naturalisme magique. On cherche à empêcher, par le biais de l'image, la dispersion de la force vivante, par exemple celle des rois, et on s'emploie à la retenir, à la fixer.

On retrouve dans les statues syro-hittites dressées à la verticale, économes de formes, le vieux motif phallique du pilier. Tammuz était à l'origine représenté comme un arbre. Le pilier est le symbole de la procréation et de la perpétuation du peuple; car l'art a ici pour rôle de préserver la force vitale de la mort assassine. Des mouvements, des dynamismes sont dès lors ignorés, des signes d'action mais aussi de rapide caducité. L'être doit obtenir des œuvres d'art durables un surcroît de force.

Pour que la statue soit efficace d'un point de vue magique, elle doit prendre forme à l'intérieur d'un rituel formel bien établi, d'un canon. Par suite, les modifications subjectives sont proscrites comme une hérésie; dans cet art, le vain bavardage des variantes à peu de frais fait entièrement défaut. Une modification de la forme standard ne peut être justifiée que par un changement de doctrine religieuse; elle revient donc à l'initiative des prêtres. La création d'images est une composante du culte et l'artiste travaille comme médium des prêtres et des princes.

Ces statues sont davantage que de pures images, ce sont des esprits vivants; l'orant projette en elles ses désirs et son énergie. Dans la haute Antiquité, on considérait que la personne était attachée à la forme plastique du corps. On peut donc parler d'une plusvalue vitale de ces statues; car en elles réside, mystérieusement, le mana du dieu ou du souverain qui est l'égal d'un dieu. La statue vit.
L'artiste travaille pour servir la commande des prêtres, il est leur médium. Les théologiens définissent le canon, et le rite des formes est répété avec humilité; car ces artistes sont soumis, dans leur création, aux principes des dieux et des temples. Pour que la statue possède une force magique, pour que le miracle attendu puisse émaner d'elle, il faut lui donner une forme fidèle au canon. Un style dictatorial et qui possède par là même une validité collective.

C'est précisément ce dogmatisme de l'Asie Mineure qui suscita chez les premiers philosophes grecs scepticisme, sarcasme et révolte. Aristophane l'Ionien le tourne ainsi en dérision: "Si les bœufs peignaient leurs dieux, ils peindraient des bœufs, et les chevaux les sculpteraient à l'image des chevaux. » Héraclite est moqueur : "Et ils priaient ces effigies des dieux comme s'ils s'entretenaient avec des maisons ${ }^{2}$." Démocrite revient d'un voyage en Asie en déclarant : « Je voudrais mieux découvrir un seul rapport de causalité que devenir roi des Perses ${ }^{3}$.» On substitue alors aux dieux les éléments délimités par le concept, et on réagit contre l'anthropomorphisme, y compris celui des Grecs; le cas échéant, on peut voir dans cette philosophie ancienne une régression vers les forces animistes, et constater son archaïsme manifeste.

Pour les hommes d'Asie Mineure, en tout cas, l'art et le savoir ne valaient qu'au sens où ils permettaient de se rapprocher des dieux qui avaient créé l'un et l'autre.

Le style hymnique, apparenté au précédent, se retrouve dans la poésie de la haute Antiquité. Au parallélisme des formes répond le strict parallélisme des métaphores. En sautant d'une image à une autre, on métamorphose symboliquement les dieux et les signes; la richesse des symboles propres au dieu témoigne de son champ d'action.

Nous analyserons ici brièvement quelques caractéristiques de la poésie de la haute Antiquité. Là encore, le style et la construction sont déterminés par un parti pris antinaturaliste. L'un et l'autre, en effet, comme la sculpture, appellent et invoquent les dieux, les forces magiques et les morts et sont par là même déterminés par des visions; en ce temps-là, en effet, le ciel et le monde des morts étaient considérés comme la réalité la plus assurée, mieux établie que l'existence terrestre du quotidien.

$\mathrm{Au}$ parallélisme des formes plastiques réglées répondait la symétrie des symboles et des métaphores dans des hymnes et des poèmes qui, comme les colon- 


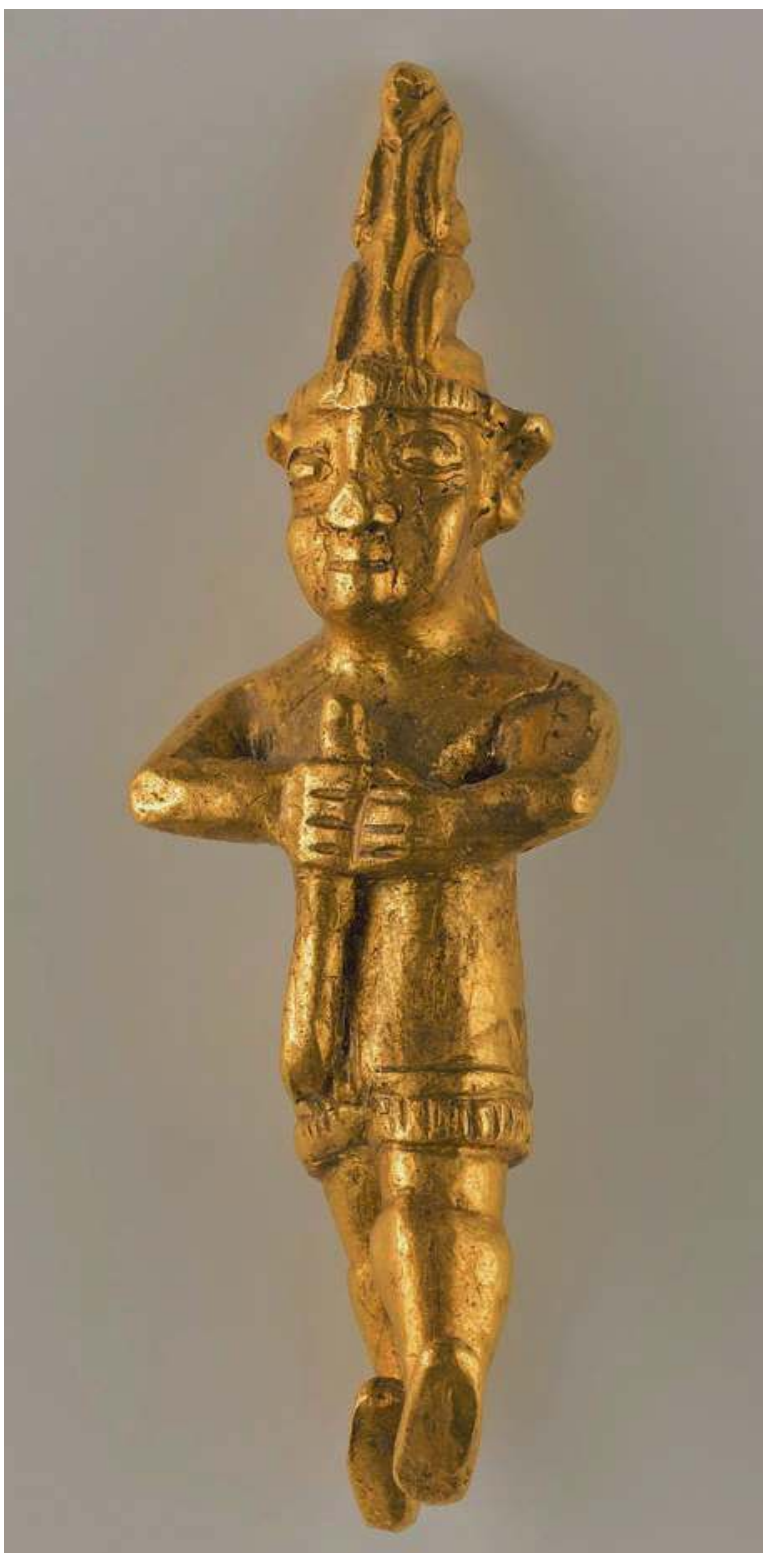

Fig. 6 Pendeloque : petit dieu hittite, Yozgat, Anatolie, vers 1500 av. J.-C., Paris, musée du Louvre @ RMN / Franck Raux.

nes, se succédaient les uns aux autres pour porter le sentiment des croyants. Dans l'apostrophe au dieu, on accumulait et additionnait les métaphores; le dieu se métamorphosait de symbole en symbole et, dans le symbole, il faisait la preuve de son efficacité sans limite sur les astres, les animaux, la terre et la mer. Les symboles recelaient le cosmos des métamorphoses du dieu et du souverain; et peut-être cette symbolique fut-elle le premier jeu de masques, la forme ancienne de la fuite devant l'identité morte.

Dans le symbole, on évite de nommer les noms divins, ces doubles que l'on redoute, et on a recours à la métaphore protectrice. Dans la symbolique, on accroît la réalité des dieux en enrichissant de forces et d'objets nouveaux leur sphère magique et en élargissant celle-ci. Le dieu révèle sa puissance active dans le drame des métamorphoses. C'est contre un tel état d'esprit que réagirent plus tard les Grecs, plus rationalistes, en posant des limites par le biais d'anthropomorphismes; c'est Socrate, c'est Sophocle proclamant qu'il "n'existe rien de plus puissant que l'homme" ou ce sont les sophistes faisant du sujet la mesure de toute chose qui tracèrent la frontière décisive séparant la Grèce de la symbolique de l'Orient, qui unifiait tout par la magie. Dès lors, les signes anciens s'étiolent dans des allégories faibles et didactiques. La langue perd sa force d'évocation immédiate et l'hymne se meurt dans le poème décoratif et les images ornementales.

Pour approcher cet homme de la haute Antiquité, il faut se souvenir que le calcul était pour lui l'exception et que le savoir passait pour une vision garantie par les dieux. Peut-être avait-on recours aux symboles pour que le dieu menaçant n'apparût pas en personne, et ils furent peut-être, par conséquent, le produit d'un tabou ancien; car en nommant le nom, on évoquait la présence corporelle de l'être redouté, le nom était son double. Peut-être ces symboles renfermaient-ils des manifestations anciennes des dieux, ou bien le dieu personnifié était-il identifié, dans le symbole, avec les puissances vénérées à des époques antérieures. Dans tous les cas, on ne peut que parler d'un style antinaturaliste et métamorphique. Peut-être un reste d'animisme est-il encore à l'œuvre dans ces symboles, l'idée, en l'occurrence, qu'une force magique est enfermée dans chaque objet et agit en lui, mais qu'une telle force doit s'écouler d'une figure à l'autre. Ainsi, on concentre dans la divinité vénérée, ou dans le souverain, un maximum de pouvoir magique en lui prêtant les symboles efficaces. Une telle poésie est étrangère à tout rationalisme. Ce qui vaut ici, ce n'est pas la réflexion, mais le saut extatique qui fait passer du signe au symbole hétérogène. Ce n'est pas la continuité causale qui est valorisée, mais l'amplitude de l'éventail des visions figurées (peut-être n'est-il pas tout à fait inapproprié de rappeler ici le principe d'exogamie, c'est-à-dire la coutume d'acquérir un supplément de force magique étrangère en épousant un membre d'une autre tribu). Cette poésie est donc identique à la tension des symboles. Chez les Grecs, la symbolique ne tarde pas à dégénérer en allégorie inoffensive, elle perd sa force d'évocation et elle est déterminée par des considérations rationnelles. Le symbole signifie l'existence des forces 
magiques dans les choses. Mais il se réduit bientôt à un ornement poétique et végète comme une ombre dans les comparaisons décoratives de ceux qui ne croient plus et se contentent d'images.

La sculpture de l'Asie Mineure syrienne comme la sculpture égyptienne sont ancrées dans des systèmes religieux qui semblent développés de façon plus conséquente que les croyances grecques. Surtout, ces religions possédaient une force dogmatique importante. Ce n'est que plus tard que les Grecs accédèrent à une systématicité contraignante dans la constitution des concepts philosophiques; les dieux, moribonds, n'étaient plus alors que des ombres et des allégories, et c'est le concept qui fut le véritable héritier du pouvoir mythique de ces divinités en déclin. Les physiciens dépassèrent alors la doctrine théologique pour mettre en place l'examen expérimental de la nature et ils interrogèrent les faits et les processus individuels. C'est grâce à cette attitude intellectuelle que les classiques grecs parvinrent à représenter la personne dans son apparence concrète et le mouvement sous des formes différenciées. L'individu vivant refoula dès lors le type formel tectonique. La personne était devenue la norme précieuse et les grands styles s'effondrèrent au contact de cette mentalité nouvelle; en effet, rien ne me paraît plus naïf que la croyance selon laquelle tous les courants d'une culture suivent un cours unifié. L'esprit analytique de la modernité grecque brisa la grande forme qui s'était développée sur la base des croyances magiques orientales.

Il s'agit à présent de replacer la haute Antiquité tectonique dans l'ensemble de l'histoire. Une telle tentative conduit à une périodisation historique dans laquelle l'art grec se voit attribuer le rôle d'une époque tardive.

Nous étudierons d'abord brièvement les bronzes hittito-syriaques. On les désigne sous l'appellation de hittites par référence à un groupe de peuples associés à la culture de l'Asie Mineure syrienne et de la Mésopotamie du Nord. Ces tribus étaient installées entre les côtes d'Asie Mineure et le haut Euphrate. Au sud du Taurus, on rencontre la culture hittite jusqu'à Qadech, au bord de l'Oronte. Les Hittites avaient connu une forte expansion vers le sud et ils s'étaient avancés loin dans le pays de Canaan. Voilà pourquoi on peut lire dans le dixième chapitre de la Genèse : "Canaan a donné naissance à Sidon, son premier-né, et à Heth.» La Bible nous apprend également qu'Abraham acheta aux Hittites la grotte de Marpela pour y placer la tombe de son épouse Sarah. On peut en déduire que les Hittites étaient présents au pays de Canaan entre 2000 et 1000 av. J.-C.

Dans ces bronzes, nous reconnaissons des éléments hittites mêlés à des caractéristiques stylistiques syrophéniciennes et égyptiennes. Une parenté manifeste les rattache à la tête de sphynx de Boghazkoï (la capitale du pays de Hatti) et à la statuette du dieu solaire de Berlin. On peut également observer les relations étroites qui unissent ces bronzes à deux sceaux-cylindres hittites, la scène d'adoration de la Morgan Library et la scène cultuelle représentée sur un sceau-cylindre des musées de Berlin. Le sceau-cylindre de la Morgan Library figure une scène d'adoration du dieu de l'orage Teshub que nous retrouvons sur quelques bronzes de la collection Stora; le dieu du sceau-cylindre de Berlin correspond aux statuettes de la même collection avec des personnages assis.

Dans cet ensemble significatif, nous observons différents types statuaires et différents stades stylistiques, depuis le style primitif strict jusqu'aux repré-

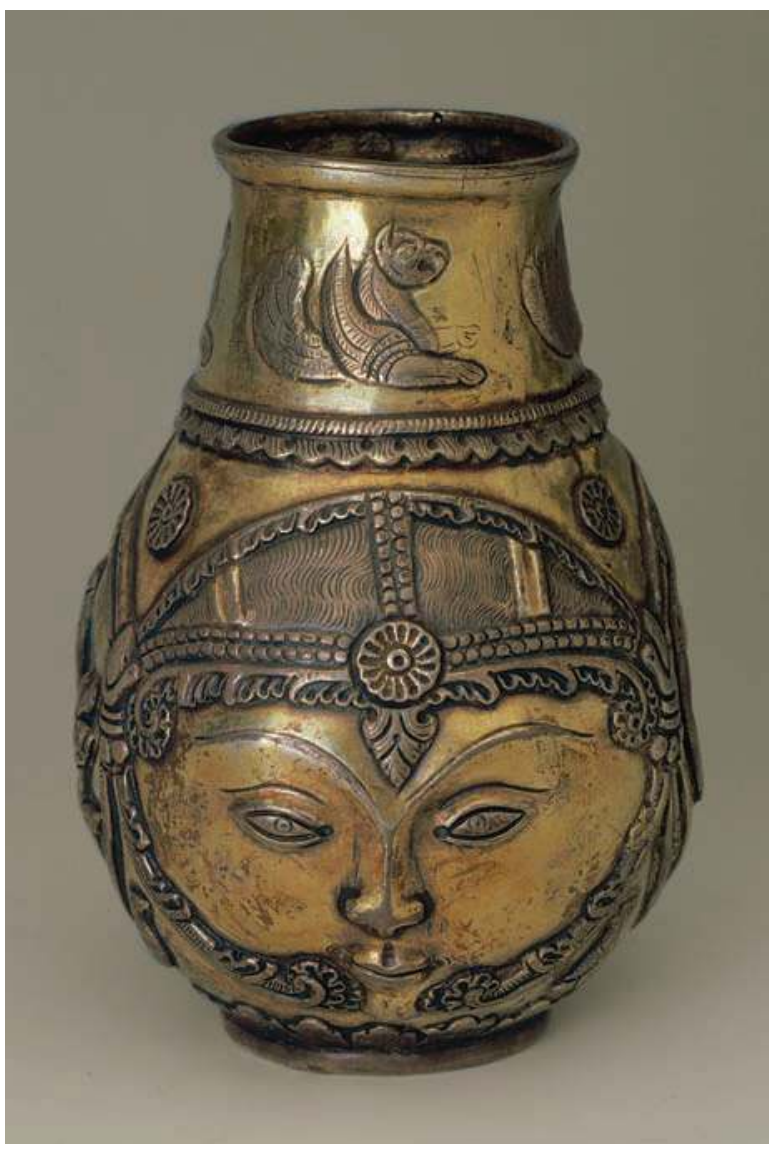

Fig. 7 Petite cruche, visage de femme et deux scènes, vi-vII ${ }^{\mathrm{e}}$ siècle, musée de l'Ermitage, Saint-Pétersbourg @ The State Hermitage Museum. Photo Vladimir Terebenin, Leonard Kheifets, Yuri Molodkovet. 
sentations animées de Teshub ou Reshef, clairement influencées par les bronzes égyptiens de l'époque du règne des Hyksos et des débuts du Nouvel Empire. Ces fusions de motifs furent conditionnées par l'introduction de conceptions égyptiennes dans la religion syrienne et la religion hittite. Les Hittites empruntèrent la conception du ciel soutenu par les dieux où plane le soleil ailé. De même, le culte des morts égyptiens exerça sans doute une influence sur les Hittites. Teshub fut souvent identifié par les Égyptiens au dieu Setesh. Les deux divinités étaient des dieux de l'orage et des intempéries. Dans le même temps, des statuettes égyptiennes et hittites représentaient le dieu des combats Reshef, semble-t-il d'origine syrienne. Il portait un bouclier et une lance. Il semble que Teshub ait ensuite plus ou moins été identifié à Reshef. Nous retrouvons un autre exemple des ces fusions de divinités dans le contrat hittite conclu par Ramsès II avec Hattushil dans sa vingt et unième année de règne: dans ce texte, des dieux hittites étaient désignés sous les noms de dieux égyptiens, par exemple Rê (le dieu du Soleil) et Setesh.

Dans la collection Stora figurent des pièces d'allure si primaire qu'on doit les dater bien avant le règne des Hyksos ou le Nouvel Empire. Les attitudes élémentaires adoptées sur ces statuettes attestent qu'elles sont nettement plus anciennes que les représentations figurées de Karkemish ou Sendshirli; elles sont également de facture plus primitive que la tête de sphynx de Boghazkoï ou que le dieu solaire de Berlin.

Différentes hypothèses pourraient justifier ce caractère primitif :

1. Le royaume hittite fut formé et gouverné par une élite indo-européenne sans doute venue d'Asie, qui pénétra en Asie Mineure et soumit la population locale. Il est possible que des groupes indo-européens aient très tôt émigré vers le sud. On peut notamment constater que la tribu hittite des Mittani effectua une telle translation. Peut-être ce déplacement s'opérat-il si tôt que ces populations s'installèrent au sud du Taurus en étant relativement peu influencées par la culture mésopotamienne plus avancée. L'hypothèse d'une migration précoce expliquerait peut-être une certaine parenté des bronzes animaliers du Mittani avec les types caucasiens. Peut-être la migration des Mittani permit-elle de contenir l'avancée des Hittites contre Babylone. Dans tous les cas, les rois des Mittani tout comme leurs dieux portaient encore des noms indo-européens; et les noms de Mithra, Varuna et Indra sont mentionnés dans les documents contrac-

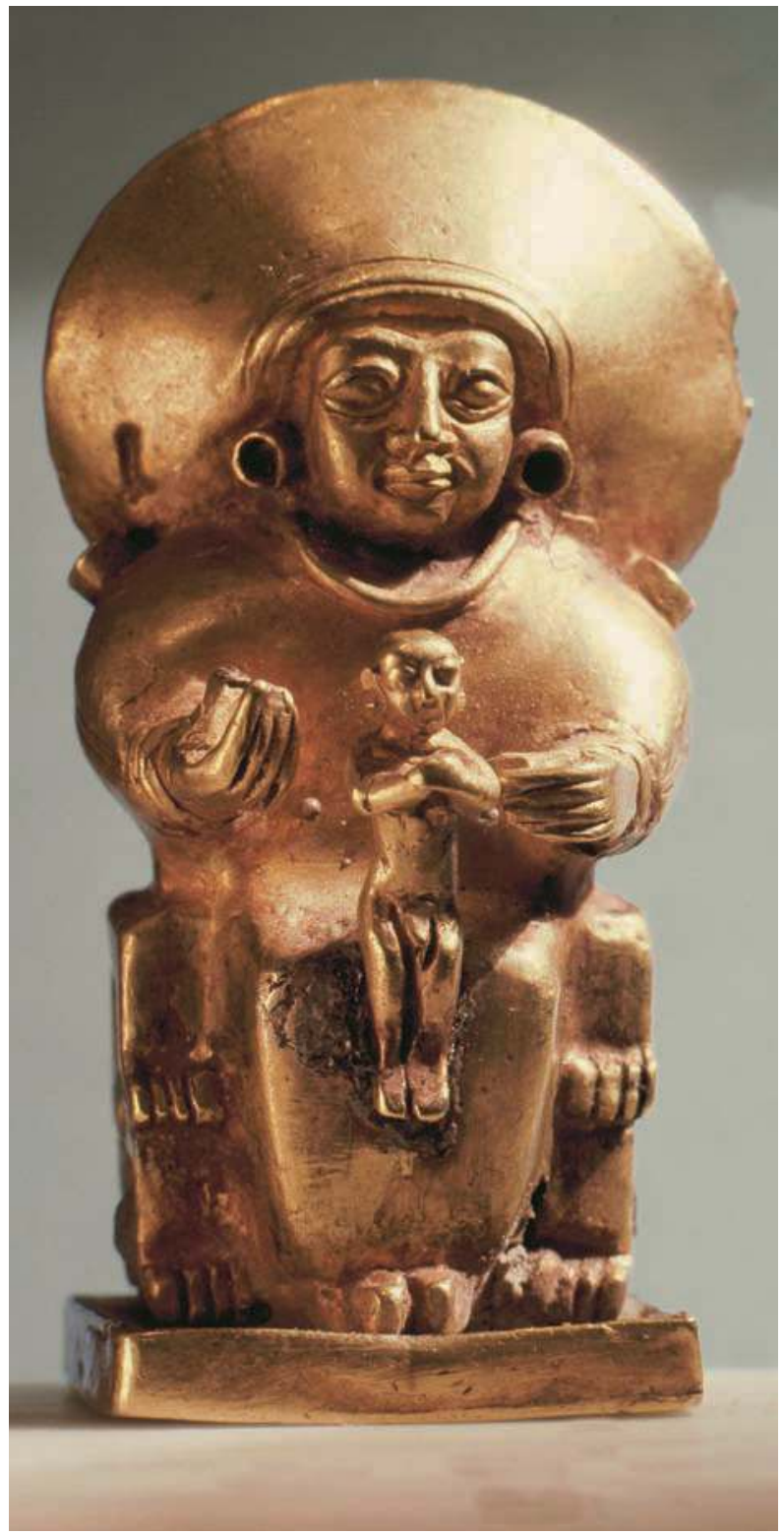

Fig. 8 Déesse assise avec enfant, XIV-XIII siècle av. J.-C., empire hittite, Anatolie centrale, New York, The Metropolitan Museum of Art, don de Norbert Schimmel Trust, 1989 / Werner Forman Archive.

tuels de Boghazkoï. Peut-être les Hyksos de Syrie s'avancèrent-ils ensuite vers l'Égypte sous la pression des conquérants indo-européens. La migration hittite ne fut qu'une vague dans le flot des migrations indoeuropéennes.

2. Une autre hypothèse pourrait éventuellement expliquer le caractère primitif de ces statuettes. Des tribus nomades non civilisées, venues du nord du désert d'Arabie, ne cessaient d'envahir les groupes désormais installés en Syrie. Un de ces groupes de nomades sémites devait jouer un rôle particulière- 
ment important en Syrie et en Palestine: les Araméens. C'est la raison pour laquelle on qualifie parfois ces sculptures d'araméennes. Des inscriptions araméennes de 1400 av. J.-C. font mention de combats contre l'Alahe Amaria, c'est-à-dire les nomades araméens qui s'avançaient vers le moyen Euphrate. Vers 1200 ou 1100 av. J.-C., ils formèrent des États en Mésopotamie et Parapotamie.

Selon nous, une part considérable de ces bronzes peut être datée d'avant l'invasion araméenne en Syrie. Entre 1200 et 1100, l'influence hittite en Syrie était déjà très affaiblie; dès lors, les Araméens contrôlèrent l'intérieur des terres syriennes depuis Sendshirli jusqu'au Jourdain oriental. Ils furent ensuite soumis durant un temps par le roi David. Plus tard, on connaît différentes principautés urbaines araméennes, en particulier le royaume de Damas.

3. L'ensemble stylistique d'Asie Mineure fut confronté à un art syro-cananéen (voir la statuette de divinité de bronze trouvée à Taanak). Il est probable que l'art d'Asie Mineure et l'art égyptien aient alors exercé une forte influence sur la culture cananéenne. Songeons par exemple à la statue assise retrouvée à Mishrif, ou encore à la tête de basalte de Djabbul, qui trahissent l'une et l'autre manifestement une influence égypto-hittite; avant tout, l'influence du Nouvel Empire.

Les relations entre les Hittites et les Égyptiens sont attestées par les tablettes d'argile de Boghazkoï et par les Lettres de Tell el Amarna. On peut se réjouir que soit présentée, dans cette exposition, une statuette de bronze de Thoutmosis III (XVIII dynastie), qui avait combattu victorieusement les Hittites et les Syriens. Les inscriptions nous apprennent que, dans la trentetroisième et la quarantième année de son règne, il reçut des cadeaux des princes de Hatti. Sur une tombe de l'époque de Thoutmosis III, on voit des envoyés syriens lui apporter des présents; les princes de Hatti et les Keftiu sont également représentés. Ces derniers étaient sans doute des Crétois minoens qui s'étaient peut-être mêlés aux Hittites sur la côte orientale de la Méditerranée.

4. Nous renvoyons encore aux relations étroites qui unissaient les sculptures hittite et syro-phénicienne. Tout comme la sculpture syro-hittite, celle des Phéniciens fut influencée par le modèle égyptien. À Göttingen sont conservées des statuettes phéniciennes qui reprennent à la lettre le motif d'Hathor.

Cette identification des divinités est attestée dans les Lettres de Tell el Amarna ainsi que dans une inscrip- tion de Ramsès II. Peu à peu, l'Astarté phénicienne était devenue si familière aux Égyptiens qu'un temple lui fut dédié en Égypte. On disait d'elle : "Vois Astarté installée dans une ville au bord de la mer, la fille de Ptah, la déesse furieuse et terrible. Les grands l'ont vue et se sont levés devant elle. Les petits l'ont vue et se sont jetés à terre. On lui a donné un siège et elle s'est assise."

5. D’autres éléments spécifiquement méditerranéens peuvent être observés sur ces statuettes. La collection Stora comporte un bronze très ancien d'origine anatolienne qui a été retrouvé à Chypre. Les bronzes aux visages ovales aplatis rappellent les idoles grecques cycladiques. Cette parenté entre la sculpture hittito-syrienne et celle de Chypre devient particulièrement patente avec les quelques statuettes de terre cuite de Karkemish, plus tardives. Les figures de plomb troyennes et les idoles égéennes et achéennes attestent le mélange profond de l'art d'Asie Mineure et de l'art grec et égéen. Ces liens particulièrement intimes se sont peut-être noués en Crète et à Chypre, notamment avec le développement important de la navigation. Cependant, l'influence de la sculpture syrienne se fit sentir jusqu'à Olympie et Delphes. On retrouve le polos et la ceinture armée hittite dans les bronzes d'Olympie.

Les Crétois et les Chypriotes, ainsi que les Mycéniens, séjournèrent sur la côte syrienne, et les voyages maritimes permirent de nouer des liens importants entre le continent, la Syrie et l'Asie Mineure. Des artistes crétois s'installèrent en Phénicie. Vers 1400, un prince syrien fit décorer un vase d'argent par des Crétois de Syrie. Au début du XIv siècle, on les retrouve à Byblos, et l'importation de céramique égéenne en Syrie rencontra un tel succès que les potiers syriens commencèrent à copier les modèles égéens.

Avec l'arrivée de nouveaux migrants achéens venus du nord, les groupes crétois et grecs furent repoussés vers l'est, ce qui resserra peut-être davantage encore les liens. Troie et Chypre furent par exemple des centres actifs de cette fusion entre le style gréco-mycénien et le style d'Asie Mineure.

Nous avons ainsià peu près fait le tour des influences exercées par la sphère formelle syro-hittite.

Au demeurant, la délimitation du style de cette aire formelle est rendue difficile par le fait que les régions situées au sud du Taurus et la Syrie étaient classiquement des régions de transit et qu'elles furent dans la plupart des cas le théâtre des guerres égypto-asiatiques, en sorte qu'elles connurent rarement la paix. 
Notons particulièrement la présence d'une statuette de guerrier apparentée à d'autres, de moindre importance, conservées à Berlin et à Kassel. Vers 1300, sous la XIXe dynastie, nous trouvons des représentations de guerriers hittites portant la barbe et des cheveux longs qui retombent en arrière, terminés par deux ou trois tresses. Peut-être ces figurations égyptiennes permettent-elles une datation approximative de cette pièce majeure.

Mentionnons encore deux bronzes syro-phéniciens d'une valeur insigne. Nous pouvons les rapprocher de bronzes syriens conservés en Russie du Sud, l'un à Cracovie, l'autre au Musée national de Varsovie4. Le British Museum possède une statuette du même type. Mais ces pièces furent peut-être des décors de vases. Les deux statues de la collection Stora me semblent particulièrement importantes; l'une porte le polos, l'autre une couronne à plumes. On observe à peu près la même parure de tête sur un disque de terre cuite de Phaïstos que l'on peut dater de goo environ, et qui représente des pulosati, c'est-à-dire des Philistins. On retrouve le même motif sur des monnaies sardes; on pourrait donc penser qu'il s'agissait là d'une parure propre aux peuples de la mer.

D'après des inscriptions égyptiennes, on date l'invasion des peuples de la mer vers 1200 environ. Sous la $\mathrm{XIX}^{\mathrm{e}}$ et la XX $\mathrm{X}^{\mathrm{e}}$ dynastie eurent lieu des combats opposant des Égyptiens à ces groupes de population mêlés. La guerre de Troie comme la destruction de cette cité furent certainement un épisode de cette phase. Ces peuples de la mer comprenaient des Crétois, des Sardes, des Étrusques, ainsi que des Philistins, les pulosati, qui donneront leur nom à la Palestine.

Ces deux statuettes se situent encore dans la sphère d'influence de l'art de l'Asie Mineure syrienne; elles possèdent encore les caractéristiques de la vision frontale. Nous pouvons voir dans ces bronzes le sommet du grand art de l'Asie Mineure syrienne parvenu à sa maturité.

Ces sculptures reprennent les motifs classiques de la vision du monde de la haute Antiquité. Le dieu ou le roi est représenté assis sur un trône; on voit aussi la Grande Mère à l'enfant, la Madone ancienne, la déesse classique du monde de l'Asie Mineure et de la Méditerranée, ou encore la déesse qui tient sa poitrine dans ses mains. Le dieu en marche est également représenté, un motif influencé par la sculpture égyptienne (on a retrouvé une pièce semblable à Schernen, en Prusse orientale, où il était certainement arrivé par la mer Noire). Mentionnons aussi les visages archaïques plats qui signalent des relations avec Chypre et les Cyclades; à Karkemish, on note la présence de l'idole hermaïque au visage triangulaire que nous connaissons à Troie, dans les îles et dans le continent.

Ces statuettes sont placées sur des socles. Nous ne savons pas s'il faut rattacher ces derniers à une croyance ancienne dans les dieux des montagnes ou s'ils sont destinés à protéger le dieu céleste ou le roi, principes de vie, du contact avec le monde souterrain mortifère et maudit.

L'exposition présente ensuite un choix de statuettes égyptiennes. Mentionnons en particulier une figure de pharaon des débuts du Moyen Empire, ou encore le portrait de Thoutmosis III, qui avait victorieusement combattu Syriens et Hittites entre l'Euphrate et l'Oronte. On connaît un hymne qui célèbre le siège de ce souverain belliqueux. Amon Rê, le seigneur de Karnak, lui dit: "Je suis venu pour que tu écrases les princes de Palestine. Je les allonge sous tes pieds à travers tes pays. Je leur montre ta majesté de Seigneur des Étoiles; tu brilles comme mon reflet devant leur face. Je suis venu pour que tu écrases le pays oriental. Tu marches sur ceux qui habitent les régions où le soleil se lève. Je leur montre ta majesté d'étoile Seshed qui sème sa flamme ardente quand elle donne la rosée. Je suis venu pour que tu écrases le pays occidental. Keftiula [la Crète] et Isi [Chypre] frémissent à ta vue, je leur donne à sentir ta puissance. Je leur montre ta majesté de jeune taureau hardi, aux cornes pointues, qui ne plie pas. [...] Je leur montre ta majesté d'oiseau [de faucon].»

Énumérons brièvement les caractéristiques des bronzes animaliers. Ceux qui proviennent de Syrie sont peut-être des bronzes ornementaux. Peut-être ces images d'animaux sont-elles aussi des substituts de sacrifice. Malgré toutes les théories du totémisme, nous ne connaissons toujours pas d'explication satisfaisante de la zoolâtrie. Peut-être est-elle un reste de l'angoisse de l'homme mal armé face à l'animal plus fort que lui; peut-être aussi une expiation du meurtre du gibier par le chasseur. On fait en sorte d'apaiser l'esprit de l'animal qu'on a tué et, peu à peu, celui-ci est divinisé. On connaît ces singulières identifications entre des dieux, des souverains et des animaux.

Une brève remarque sur les bronzes animaliers égyptiens. À l'époque saïte, le culte des animaux ressurgit avec une intensité inhabituelle; une curieuse régression. Peut-être l'ancienne religion populaire ou les cultes astraux qui avaient été refoulés par les cultes officiels des cours royales et des temples et par la théo- 


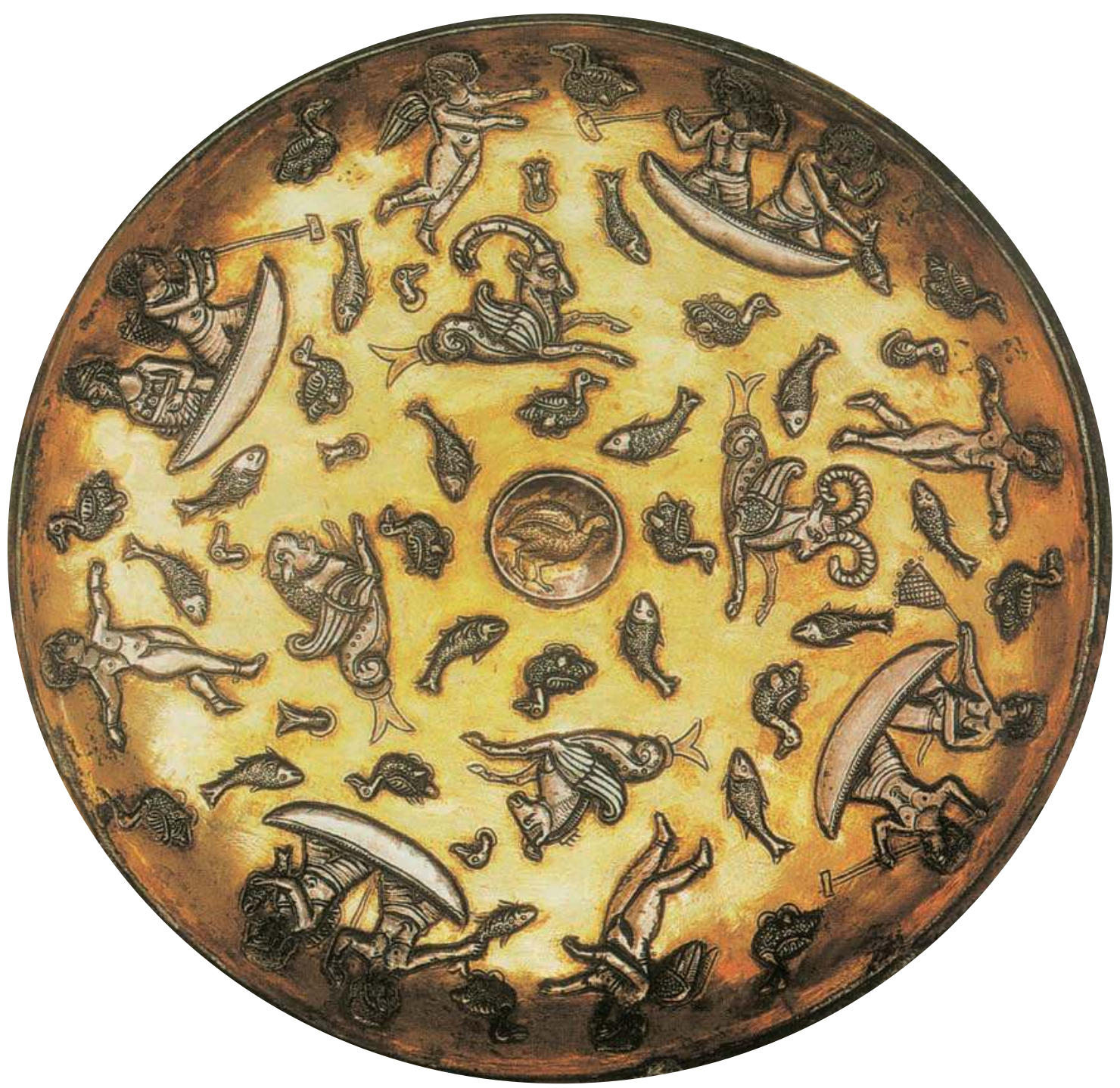

Fig. 9 Plat, décor de pêcheurs, Iran, Rashi (Gilan), vI-VII ' s., Téhéran, Musée national.

logie des prêtres reviennent-ils en force à la faveur de la dissolution de l'empire et de la société. L'adoration des animaux exprime peut-être une intuition de l'unité mythique de l'univers. Peut-être adore-t-on l'animal parce qu'il est plus proche de la nature mystérieuse et de ses éléments magiques. Dans tous les cas, on peut voir dans le culte animalier des Égyptiens saïtes un singulier archaïsme religieux. Cet état d'esprit a été conservé dans une chanson à boire de l'époque, qui décrit le bonheur des morts et qui est typique de l'état d'esprit des Égyptiens tardifs et de leur piété archaïque. Le harpiste chante : «J'ai entendu les chants qui sont inscrits dans les tombes des temps anciens. De quoi parlent-ils quand ils célèbrent la vie sur la terre et font peu de cas de la cité des morts? [...] À quoi bon agir ainsi à l'encontre du pays de l'éternité? Ce pays qui n'a pas d'ennemis; tous nos parents reposent en lui depuis les premiers temps, et tous ceux qui naissent reposeront en lui. Nul n'a pu rester en Égypte et il n'est personne qui n'ira dans ce pays. Le temps de notre séjour sur la terre est une vision de rêve.»

Le présent ne vaut donc rien au regard de l'histoire et de l'éternité des morts; voilà pourquoi on imite avec lassitude les images des anciens.

Le caractère sacré des animaux est particulièrement enseigné dans l'Égypte ancienne, et c'est cette croyance qui a donné naissance à un grand nombre de bronzes animaliers. Cette adoration des animaux traduit une réaction contre la religion spéculative des théologiens. 
Mentionnons encore, dans ce contexte, les deux doubles haches crétoises (labris) qui sont certainement des insignes du taureau cornu, des symboles des divinités solaires.

Arrêtons-nous brièvement sur quelques pièces encore. Tout d'abord le bronze béotien qui représente un paysan en train de labourer avec des taureaux (vi ${ }^{e}$ siècle). Le Louvre possède un modèle semblable en terre cuite, et on trouve un autre exemple de même type au British Museum. Cette sculpture archaïque paysanne est tout à fait dans l'esprit des Erga Kai Hemerai d'Hésiode5. Le berger Hésiode, l'homme de la province conservatrice, me semble avoir préservé l'esprit de la Grèce ancienne sous une forme plus pure que les poèmes homériques. Ce bronze est pour nous comme une illustration des vers d'Hésiode. Nous citerons par conséquent le poète de Béotie, qui invite son frère Persée à travailler la terre : "Telle est la loi des champs, et elle vaut aussi bien pour ceux qui habitent les côtes des mers que pour ceux qui sont installés au creux des vallées, loin des vagues et des flots, sur la terre fertile. Sème nu, travaille nu, récolte nu, si tu veux mener à bien quand il le faut les travaux de Déméter ${ }^{6}$. Ce petit bronze résume sous une forme stricte et condensée le chant consacré par Hésiode aux travaux des champs.

Une partie importante de l'exposition est occupée par une série de bronzes étrusques. Des groupes de cette ethnie ont certainement fait partie des peuples de la mer que combattirent les Égyptiens du Nouvel Empire. Selon la plupart des traditions anciennes, ils venaient de l'est et l'inscription de la stèle de Lemnos fut peut-être un témoignage de leur migration. Ils semblent s'être introduits en Italie par le sud-ouest.

D'après Hérodote, ils venaient de Lydie; les Grecs les appelaient les Tyrrhéniens; on les connaît encore sous l'appellation égyptienne de Tursha. La tradition égyptienne rapporte que, aux xiI et xiII ${ }^{e}$ siècles, avec d'autres peuples "des côtes maritimes les plus lointaines", ils étaient venus dans le delta du Nil pour y pratiquer le pillage. Peut-être ces populations avaientelles fui devant la jeune puissance achéenne en se déplaçant vers l'ouest, vers l'Italie. La stèle de Lemnos indique que la côte asiatique fut un point de départ.

Les Étrusques sont les véritables colonisateurs de l'Italie, et c'est d'eux que sont hérités les éléments les plus nobles de l'éclectisme romain. Dans tous les cas, ce sont eux qui, avec les Grecs, ont forgé les impulsions fondamentales de la culture romaine. Pourtant, la tradition des Latins passe obstinément sous silence cet héritage majeur, avec une suffisance de parvenus.

Rome doit son urbanité aux Étrusques qui avaient rassemblé les villages des sept collines en une ville, etrusco ritu, et l'avaient entourée d'un mur; ce sont les Étrusques, en effet, qui avaient créé une culture urbaine dans l'arrière-pays italien. Pourtant, ce groupe isolé d'un point de vue ethnique comme d'un point de vue culturel s'usa peu à peu dans de vains combats et ne put opposer une résistance durable aux paysans et aux bergers italiques, supérieurs en nombre. Ils furent lentement éclipsés par la population indigène. Peut-être ces navigateurs étaient-ils venus en Italie avec un héritage culturel trop limité pour ne pas succomber progressivement à la fusion fatale avec les tribus indigènes.

On peut distinguer deux ensembles stylistiques dans l'art étrusque : le style orientalo-phénicien, qui dure environ de 800 à 625; par la suite, l'influence orientale, dans laquelle des composantes égyptiennes étaient également à l'œuvre, céda la place aux influences grecques. La période qui va de 625 à 475 peut être désignée comme la période archaïque grecque; les $v^{e}$ et $\mathrm{IV}^{\mathrm{e}}$ siècles correspondent ensuite au style classique. Du ${ }{ }^{\mathrm{e}}{ }^{\mathrm{e}} \mathrm{au} \mathrm{I}^{\mathrm{er}}$ siècle, des tendances hellénistiques s'affirment dans l'art étrusque. La langue étrusque disparaît progressivement, au profit de la langue latine. L'influence étrusque se perpétue surtout dans la religion et le théâtre romains. On emprunte aux Étrusques la prophétie climatique et le calendrier sacré; beaucoup d'expressions de l'art du théâtre, par exemple le masque, persona, sont d'origine étrusque. L'examen des foies est aussi une marque de l'origine orientale des Étrusques; le foie de bronze étrusque de Plaisance (un reflet du cosmos ${ }^{7}$ ) présente une ressemblance frappante avec un foie divinatoire hittite du Boghazkoï, de la même façon qu'on peut présupposer une certaine parenté entre l'art étrusque et la sculpture chaldéenne de Tushpa. Ces faits apportent sans doute la preuve que l'immigration étrusque s'effectua par vagues successives, jusqu'en 800 av. J.-C. peut-être.

Un peuple tragique, qui donna énormément à l'Italie, mais qui est pourtant systématiquement tombé dans l'oubli. L'art de ce peuple est d'une noblesse qui ne se détache jamais tout à fait de la vieille tectonique orientale, même dans la période hellénistique. On sent dans cet art l'écho lointain de couches tectoniques anciennes, et c'est précisément cette perpétuation d'un esprit archaïque qui le distingue de l'art grec. Peut-être un lointain reflet de l'esprit étrusque brillet-il encore dans la première poésie latine, notamment 
dans les élégies de Properce. À la vue de ces sculptures, on pourrait parler d'une subtile touche orientalisante, d'une nostalgie de l'Orient qui n'est jamais tout à fait recouverte par la modernité grecque et son affirmation résolue. Il est certain que les Grecs assimilèrent et absorbèrent les influences orientales d'une façon plus résolue et plus originale. Mais les bronziers étrusques n'étaient guère en retrait par rapport à eux, et nous percevons dans ces œuvres l'écho et le deuil d'une grande culture intellectuelle qui avait prédominé avant le classicisme scolaire.

Cette exposition présente des exemples de la grande culture préhellénique; notamment des œuvres de l'époque tectonique qui succéda sans doute à une époque de réalisme dramatique. L'importance de cette période de la haute Antiquité tient à la stricte sélection des formes et à la discipline de construction qui furent les siennes. C'est à cette époque que furent fixés les motifs fondamentaux de la création plastique; l'Antiquité ne fit ensuite que produire des variations ou dissoudre la forme condensée dans un trop-plein phraséologique. Cet art tectonique nous apparaît très humain parce que l'homme se défend ici contre le vertige des innombrables variantes de l'expérience en affirmant des formes typiques, sacrées et dotées d'une efficacité magique, et en créant un ordre. Ces formes ont force de loi; voilà pourquoi elles reviennent sans cesse. Telle fut la leçon que les Grecs [du viII ${ }^{\mathrm{e}}$ et du $\mathrm{VII}^{\mathrm{e}}$ siècle tirèrent de la grande école de l'Orient; le haut Moyen Âge, lui aussi, hérita de l'Orient un sens de la grande forme. La forme originelle nous préservera toujours du vertige et de l'inessentiel, car elle est une projection de l'origine plastique primitive, bâtie sur le corps humain, tout comme l'espace acquiert une forme et une signification parce qu'il est une expression de l'énergie humaine. La forme arrondie de la tête, la surface elliptique du visage, le cube du torse, les piliers cylindriques des jambes sont des prototypes; ces premiers motifs furent découverts et développés par des hommes de la haute Antiquité en accord avec les lois vivantes du corps humain ${ }^{8}$.]

- $\bullet$

1. Cf. Liliane Meffre, Carl Einstein (1885-1940). Itinéraires d'une pensée moderne, Paris, Presses de l'université Paris-Sorbonne, 2002, p. 256.

2. Héraclite, Fr. 5.

3. Démocrite, B 118.

4. L'appellation «Russie du Sud » pour la Pologne n'était pas usuelle en Allemagne à l'époque.

5. Les Travaux et les Jours (début du vil ${ }^{\mathrm{e}}$ siècle av. J.-C.).

6. Ibid., vers 388 à 394.

7. Le foie de bronze étrusque retrouvé à Plaisance, en Italie (fin du $\|^{\mathrm{e}}$ ou début du $\mathrm{I}^{\mathrm{er}}$ siècle av. J.-C.], portait, dans sa partie convexe, une figuration du cosmos des divinités séparées en cases; un lien était ainsi établi entre hépatoscopie et cosmologie ( $c f$. Giovannangelo Camporeale, in Les Étrusques et l'Europe, catalogue d'exposition, Paris, Réunion des Musées nationaux, 1992, p. 80).

8. Le passage final entre crochets, dont la page a été perdue dans le manuscrit original allemand, a été traduit à partir de la version anglaise du texte. 